\title{
Uncertain Time Series Classification
}

\author{
Michael Franklin Mbouopda \\ Clermont Auvergne University, CNRS, ENSMSE, LIMOS, F-63000, Clermont-Ferrand, France \\ michael.mbouopda@uca.fr
}

\begin{abstract}
Time series analysis has gained a lot of interest during the last decade with diverse applications in a large range of domains such as medicine, physic, and industry. The field of time series classification has been particularly active recently with the development of more and more efficient methods. However, the existing methods assume that the input time series is free of uncertainty. However, there are applications in which uncertainty is so important that it can not be neglected. This project aims to build efficient, robust, and interpretable classification methods for uncertain time series.
\end{abstract}

\section{Motivation}

Time series analysis has gained a lot of interest during the last decade. In fact, time series are used in many applications such as smoking and sleep detection [Adams and Marlin, 2018], outlier detection detection [Kieu et al., 2019] and astronomical objects classification [Allam Jr et al., 2018]. The field of time series classification has been particularly active recently with the development of more and more efficient methods [Ruiz et al., 2020; Bagnall et al., 2017]. However, the existing methods assume that the input data is free of uncertainty. This assumption does not hold in every application. For instance, the Plasticc dataset [Allam Jr et al., 2018] contains time series representing astronomical objects such as galaxies and stars. The measurement method produces time series that are not free of uncertainty. Fig. 1 shows a sample from the Plasticc dataset. The blue line is the best guess of the time series and the red region represent the uncertainty region. Any time series that lies in the red region could be the exact (unknown) time series.

More generally, imprecision in data can be caused by the environment, the collection method and technique, privacy constraints and other causes. This imprecision adds epistemic uncertainty in the collected data, and in some applications, it is important for machine learning models to take that into account in order to be efficient.

In order to apply the existing methods to uncertain data, one approach is to simply ignore uncertainty. This approach might work when the uncertainty is not important and can be neglected. The second approach is to remove uncertainty

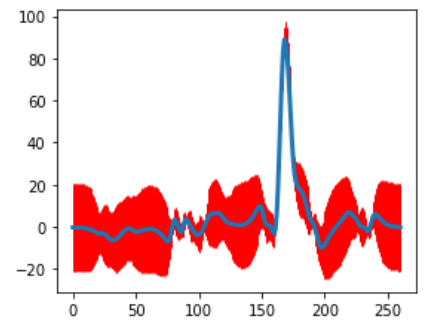

Figure 1: An uncertain time series from the Plasticc dataset

from the data using a preprocessing technique. This approach requires domain knowledge which is not always available. The last approach is to use a method that can automatically handle uncertainty in the data. We use the term end-to-end to qualify such a method. An end-to-end method can be used efficiently without any domain knowledge, since it learns by itself how to handle uncertainty. The field of uncertain time series analysis is still under-explored and the goal of this research project is to build end-to-end methods for uncertain time series analysis.

\section{Project Description}

Before giving the specific goals of this research project, let us define some core notions.

Definition 1 (Time series). A time series $T$ of length $m$ is an ordered sequence of $m$ observations $t_{i}$.

$$
T=<t_{1}, t_{2}, \ldots, t_{m}>, t_{i} \in \mathbb{R}
$$

Definition 2 (Uncertain time series). An uncertain time series (uTS) is a time series in which the observations are uncertain.

There are two models for representing an uncertain observation:

- the multi-set based model represents each uncertain observation by a finite set of real values. That is $t_{i}=$ $\left\{t_{i 1}, t_{i 2}, \ldots, t_{i k}\right\}, k \in \mathbb{N}$

- the probability density function (PDF) based model represents each uncertain observation by a mean and a deviation. That is $t_{i}=\hat{t_{i}} \pm \delta t_{i}$, meaning that the unknown exact value of $t_{i}$ belongs the interval $\left[\hat{t}_{i}-\delta t_{i} ; \hat{t}_{i}+\delta t_{i}\right]$ and follows a particular probability distribution. 
Definition 3 (uTS classification task). Given a dataset $D=$ $\left\{<T_{1}, c_{1}>,<T_{2}, c_{2}>, \ldots,<T_{n}, c_{n}>\right\}$ of $n$ uncertain time series $T_{i}$, each with its class label $c_{i}$ taken from a discrete finite set $C$, the task of uTS classification consists of learning a function that maps the uTS to their class labels.

The goal of this research project is to build end-to-end methods that can resolve the uncertain time series classification task. The methods must have the following properties:

- Efficiency: The methods should produce accurate and reliable predictions

- Robustness: The methods should be robust to noise and to uncertainty variation in the data. This is very important since we know that the existing time series classifier are vulnerable to adversarial attacks [Fawaz et al., 2019; Karim et al., 2020]

- Interpretability: Finally, the methods should provide tools that help to explain or interpret predictions.

\section{Contribution}

We have proposed the Uncertain Shapelet Transform (UST) algorithm [Mbouopda and Nguifo, 2020], an adaptation of the shapelet transform algorithm [Hills et al., 2014] for uncertain time series. UST is to our knowledge the first and only end-to-end method for uncertain time series classification. UST is built by propagating uncertainty throughout the shapelet transform algorithm. This is achieved by the use of the Uncertain Euclidean Distance (UED) which is obtained by taking uncertainty into account in the Euclidean Distance. UST has two principal components: the uncertain similarity measure used to extract features and the classifier used after features extraction. By propagating uncertainty during features extraction, the classifier can take the propagated uncertainty into account in order to build better decision boundaries: The more the classifier is aware of uncertainty, the better are its predictions. The principal UST components can be configured regarding the dataset characteristics. We shown experimentally that UST is efficient and robust to uncertainty. UST predictions can be explained by visualizing the features (which are actually patterns appearing in the time series) that are more correlated with the predictions.

\section{Perspectives}

In our paper [Mbouopda and Nguifo, 2020], we applied UST on dataset downloaded from the UEA \& UCR repository [Dau et al., 2019]. Since these datasets do not have uncertainty, we manually added synthetic uncertainty to the one we used. The first future work is to apply our method on some real uncertain datasets such as the Plasticc dataset [Allam Jr et al., 2018].

UST is based on a single type of features called shapelet. Simply said, a shapelet is a pattern that is common to time series that belong to the same class. It has been shown that there are many datasets that cannot be efficiently classified using only shapelets [Bagnall et al., 2017]. Therefore, another future work is to build methods that use different type of features.

\section{Acknowledgments}

This work is funded by the French Ministry of Higher Education, Research and Innovation and is supervised by Prof. Engelbert MEPHU NGUIFO ( https://perso.isima.fr/ $\sim$ enmephun/)

\section{References}

[Adams and Marlin, 2018] Roy Adams and Benjamin M Marlin. Learning time series segmentation models from temporally imprecise labels. In $U A I$, pages 135-144, 2018.

[Allam Jr et al., 2018] Tarek Allam Jr, Anita Bahmanyar, Rahul Biswas, Mi Dai, Lluís Galbany, Renée Hložek, Emille EO Ishida, Saurabh W Jha, David O Jones, Richard Kessler, et al. The photometric lsst astronomical timeseries classification challenge (plasticc): Data set, 2018.

[Bagnall et al., 2017] Anthony Bagnall, Jason Lines, Aaron Bostrom, James Large, and Eamonn Keogh. The great time series classification bake off: a review and experimental evaluation of recent algorithmic advances. Data Mining and Knowledge Discovery, 31(3):606-660, 2017.

[Dau et al., 2019] Hoang Anh Dau, Anthony Bagnall, Kaveh Kamgar, Chin-Chia Michael Yeh, Yan Zhu, Shaghayegh Gharghabi, Chotirat Annh Ratanamahatana, and Eamonn Keogh. The ucr time series archive. IEEE/CAA Journal of Automatica Sinica, 6(6):1293-1305, 2019.

[Fawaz et al., 2019] Hassan Ismail Fawaz, Germain Forestier, Jonathan Weber, Lhassane Idoumghar, and Pierre-Alain Muller. Adversarial attacks on deep neural networks for time series classification. In 2019 International Joint Conference on Neural Networks (IJCNN), pages 1-8. IEEE, 2019.

[Hills et al., 2014] Jon Hills, Jason Lines, Edgaras Baranauskas, James Mapp, and Anthony Bagnall. Classification of time series by shapelet transformation. Data Mining and Knowledge Discovery, 28(4):851-881, 2014.

[Karim et al., 2020] Fazle Karim, Somshubra Majumdar, and Houshang Darabi. Adversarial attacks on time series. IEEE transactions on pattern analysis and machine intelligence, 2020.

[Kieu et al., 2019] Tung Kieu, Bin Yang, Chenjuan Guo, and Christian S Jensen. Outlier detection for time series with recurrent autoencoder ensembles. In IJCAI, pages 27252732, 2019.

[Mbouopda and Nguifo, 2020] Michael Franklin Mbouopda and Engelbert Mephu Nguifo. Uncertain time series classification with shapelet transform. In 2020 International Conference on Data Mining Workshops (ICDMW), pages 259-266. IEEE, 2020.

[Ruiz et al., 2020] Alejandro Pasos Ruiz, Michael Flynn, James Large, Matthew Middlehurst, and Anthony Bagnall. The great multivariate time series classification bake off: a review and experimental evaluation of recent algorithmic advances. Data Mining and Knowledge Discovery, pages $1-49,2020$. 